\title{
Experimentando o SPIDe aplicado à Elicitação de Requisitos ${ }^{\star}$
}

\author{
Jean C. S. Rosa ${ }^{1}$, Ecivaldo Matos ${ }^{1}$, Fiama S. Santos ${ }^{2}$ e Gilton J. F. Silva ${ }^{2}$ \\ ${ }^{1}$ Departamento de Ciência da Computação (DCC) \\ Universidade Federal da Bahia - Salvador - Brasil \\ ${ }^{2}$ Departamento de Computação (DComp) \\ Universidade Federal de Sergipe - São Cristóvão - Sergipe \\ \{jean.rosa, ecivaldo\}@ufba.br, \{fssantos, gilton\}@dcomp.ufs.br
}

\begin{abstract}
Resumo A elicitação de requisitos é o momento que exige uma intensa comunicação entre os "donos de problemas" e os engenheiros de requisitos. Para elicitar requisitos é necessário que os engenheiros busquem técnicas adequadas, que favoreçam a comunicação. Uma das recomendações da literatura para facilitar a elicitação é o contato direto com os usuários, o que pode ser alcançado por meio do Design Participativo (DP), haja vista que suas técnicas podem proporcionar uma comunicação mais robusta. No Design de Interação, um processo metodológico compostou por técnicas participativas é o SPIDe. O SPIDe é um processo semioparticipativo, ou seja, associa o DP à Semiótica para estudar a comunicação por meio da participação do usuário no processo de design. Sob essa perspectiva questiona-se: é possível elicitar requisitos por meio do SPIDe? Este artigo apresenta um estudo experimental para identificar se é possível elicitar requisitos por meio da aplicação do SPIDe. A partir dos resultados gerados foi concluir que por meio do SPIDe é elicitar requisitos, permitindo que os usuários tornem-se co-autores na elicitação de requisitos do software a ser desenvolvido.
\end{abstract}

Keywords: Engenharia de Requisitos · Fatores Humanos · Engenharia Semiótica.

\section{Introdução}

A Engenharia de Requisitos (ER) é uma atividade estritamente humana e é composta por etapas de elicitação, análise, especificação e validação de requisitos [8]. De acordo com Bourque e Fairley [8], requisito é um atributo a fim de resolver algum problema e, portanto, devem expressar as necessidades e as restrições da solução que será desenvolvida. Da Costa e Pimentel [10] esclarecem que para construir/desenvolver softwares se faz necessário entender a necessidade do usuário e atender as suas expectativas, isto é, engenhar requisitos.

\footnotetext{
* Agradecemos aos participantes da pesquisa; ao Grupo de Pesquisa e Extensão em Informática, Educação e Sociedade - Onda Digital (CNPq/UFBA); e a CAPES por financiar esta pesquisa.
} 
Engenhar requisitos é um processo difícil, que não precisa ser caro [14]. Uma das principais etapas/atividades da ER é a elicitação [24]. A elicitação de requisitos é um processo para descoberta e coleta das necessidades dos usuários, dos seus desejos, dos seus problemas e de como é possível solucioná-los [16]. Para isso, é necessário uma intensa comunicação com os "donos dos problemas" ${ }^{3}$. A comunicação entre usuários e engenheiros de requisitos é um dos fatores humanos e sociais intrínsecos à elicitação de requisitos, sendo considerado como núcleo da ER [6].

Da Costa e Pimentel [10] descrevem que em algumas situações os usuários não sabem comunicar, de maneira clara, o que necessitam ou desejam. Para remediar essas situações esses pesquisadores, e outros $[25,9,16]$, sugerem que o engenheiro de requisitos busque técnicas adequadas para facilitar a elicitação de requisitos. Nesse sentido, Das [11] corrobora ao evidenciar que o contato direto com os usuário auxilia os engenheiros a estudar os reais requisitos e indica que os contatos indiretos são menos desejáveis, pois podem causar ruídos e até mesmo distorções na comunicação.

No Design de Interação, um processo metodológico que permite o contato direto entre o designer de interação e os usuários é o SPIDe ${ }^{4}$ [20]. Isso é devido a sua composição por técnicas de Design Participativo (DP). De acordo com Simonsen e Robertson [22], o DP proporciona a participação direta e efetiva das pessoas no desenvolvimento de artefatos que moldam as suas vidas, dentre eles os software. Nesse sentido, o uso de técnicas participativas podem estimular uma comunicação mais robusta e o compartilhamento entre usuários e designers de interação. Portanto, devido a sua composição, o SPIDe pode favorecer da comunicação.

Além do DP, a base teórico-conceitual do SPIDe é a Engenharia Semiótica (EngSem), uma teoria de Interação Humano-Computador. Para a teoria criada por De Souza [12], a interação (humano-computador) é um processo comunicativo entre os designers e os usuários. Por ter base semiótica, o SPIDe pode auxiliar os designers no processo de design de interação por meio do estudo da comunicação, um das principais aplicações da Semiótica. Seria então possivel elicitar requisitos por meio do SPIDe?

Nesse sentido, este artigo tem como objetivo apresentar os resultados de um estudo experimental sobre a potencialidade do SPIDe na elicitação de requisitos de software. O estudo foi realizado durante a concepção de um Ambiente Virtual de Aprendizagem (AVA) para uma Biblioteca Pública Brasileira. A concepção do AVA faz parte do projeto de extensão universitária intitulado "Uma proposta de Ambiente Virtual de Aprendizagem para disseminação da inclusão digital na sociedade Sergipana", vinculado à Universidade Federal de Sergipe.

$\mathrm{O}$ artigo está dividido em sete seções, sendo a próxima seção destinada à comunicação como um desafio para a elicitação de requisitos; a seção 3 apresenta

\footnotetext{
3 Assumi-se neste artigo como "donos do problema" os clientes, sujeitos-interessados, (potenciais) usuários finais ou partes interessadas. Será sempre utilizado o termo usuário para indicar esse grupo de sujeitos.

${ }^{4}$ Semio-Participatory Interaction Design
} 
alguns trabalhos relacionados, que tratam sobre semioparticipação na engenharia de requisitos. O SPIDe é descrito na seção 4 e a metodologia de pesquisa na seção 5. Por sua vez, os resultados e as considerações finais são apresentados nas seções 6 e 7 , respectivamente.

\section{A Comunicação na Elicitação de Requisitos}

A ER é uma atividade estritamente humana [8]. Essa característica lhe faz ser influenciada pela subjetividade dos humanos que a aplicam e pela subjetividade dos humanos que participam da ER [12]. Neste artigo, os humanos que aplicam a ER são chamados de engenheiros de requisitos.

É função do engenheiro de requisitos identificar, representar e gerenciar as perspectivas dos mais variados usuários no projeto de software. A descoberta ou elicitação dos requisitos não é uma atividade passiva, deve haver a cooperação e articulação entre os usuários e o engenheiro [8]. Para Baranauskas et al. [5], a ER não se limita a identificar, representar e gerenciar os requisitos, trata-se de um processo para facilitar a comunicação entre os engenheiros de requisitos e os usuários. Nesse sentido, a comunicação torna-se um dos princípios essenciais de eficácia da elicitação de requisitos [8,25].

A comunicação é um processo cultural, bem como a significação. Ambas, comunicação e significação, são temas de estudo da Semiótica [13]. Santaella [21] define a Semiótica como a ciência dos signos e, para a pesquisadora, signo é qualquer coisa que tenha significado para qualquer sujeito. A significação, ou semiose, é o processo de dar significado aos signos. O conjunto de signos pessoais formulados dentro de um contexto social ou cultural é chamado de sistema de significação [13].

De acordo com Eco [13], a comunicação é realizada por meio de signos, influencia e é influenciada pela cultura. Para que a comunicação seja efetiva é necessário que os interlocutores compartilhem semelhanças culturais, senão, haverá falhas na interpretação, isto é, problemas de significação. A falha na comunicação pode fazer com que requisitos não sejam elicitados ou até mesmo elicitados equivocadamente $[9,25]$.

Nesse sentido, para facilitar e tornar eficiente a comunicação entre os engenheiros de requisitos e os usuários, Baranauskas et al. [5] propõem a associação de uma abordagem semiótica para sistemas de informação, a Semiótica Organizacional, e uma abordagem participativa, o Design Participativo. Baranauskas [3] utiliza-se da expressão "semioparticipativo" para qualificar a associação entre uma abordagem semiótica e uma abordagem participativa.

Conforme a perspectiva de Baranauskas [3] sobre semioparticipação, a abordagem semiótica trata sobre a comunicação efetiva entre os engenheiros de requisitos (ou designers de interação, no caso da Interação Humano-Computador) e os usuários, considerando fenômenos físicos, psíquicos e sociais tanto dos engenheiros de requisitos, quanto dos usuários; tratando as comunicações e as significações dos sujeitos. Por sua vez, as abordagens participativas tendem a colocar os usuários como participantes efetivos na concepção de um software. Nesse 
sentido, o uso de uma abordagem semioparticipativa proporciona o estudo da comunicação entre os agentes tornada efetiva por meio de sua participação na concepção de um software.

A comunicação entre os engenheiros de requisitos e os usuários tem sido um desafio que, segundo Zin e Pa [25], ultrapassa mais de 30 anos de estudos, mas não é um tema saturado. Esse desafio tem relação com a gama de aspectos coexistentes nos processos de engenharia de requisitos, como aspectos cognitivos, culturais, contextuais, subjetivos, além do uso de técnicas e ferramentas apropriadas por parte dos engenheiros $[17,16]$. Por isso, o modo como os usuários participam da elicitação de requisitos pode ser fator determinante para a eficácia na comunicação nesse processo [17,9]. De acordo com Das [11], é indiscutível que a participação dos usuários na elicitação de requisitos proporciona efeitos positivos e melhora a experiência do usuário. Das [11] ainda alerta que a partir da participação dos usuários na elicitação de requisitos é possível diminuir a quantidade de erros nos requisitos elicitados.

\section{Trabalhos Relacionados}

Para favorecer a comunicação na Engenharia de Requisitos e a participação dos usuários, Baranauskas et al. [5] propõem o uso da Semiótica Organizacional em associação ao Design Participativo para a Elicitação de Requisitos. Para os autores, graças à essa associação foi possível identificar a facilitação no entendimento compartilhado dos requisitos, uma vez que houve um contato direto dos engenheiros de requisitos com os usuários. Por sua vez, o uso da Semiótica Organizacional proporcionou a sensibilização dos engenheiros de requisitos quanto à forma como os usuários percebem, compreendem e interagem com/no mundo ao seu redor.

A pesquisa apresentada por Baranauskas et al. [5] limitou-se ao uso do método MEASUR, da Semiótica Organizacional, e Workshops de Design Participativo. Todavia, os pesquisadores não detalharam quais técnicas participativas foram utilizadas nos Workshops.

Por sua vez, Arantes [2] também utilizou a proposta semioparticipativa de Baranauskas et al. [5]. Em seu trabalho, Arantes realiza um estudo de caso para elicitação de requisitos de um web site. Entretanto, a autora não detalha quais técnicas participativas foram utilizadas em seu estudo de caso, o que implica em dificuldades na replicação do estudo e, até mesmo, no uso da proposta participativa por outros pesquisadores ou profissionais da indústria de software.

Em um estudo posterior, Arantes [1] apresenta uma proposta semioparticipativa para quatro processos da ER, a saber: elicitação, análise, especificação e validação. Mais uma vez a pesquisadora utiliza como base o método MEASUR, da Semiótica Organizacional, e Workshops de Design Participativo. Entretanto, dessa vez fica explícito o uso das técnicas participativas brainstorm e braindraw, durante os Workshops de Design Participativo. Arantes utilizou o brainstorm para identificação dos stakeholders e para a elicitação dos requisitos. Por sua vez, o braindraw é utilizado para a construção de protótipos, depois da ne- 
gociação de requisitos. Para a autora, o processo semioparticipativo utilizado facilitou a discussão entre os usuários durante os workshops, conduzindo a uma melhor compreensão do contexto e das implicações sociais do sistema.

Sob a perspectiva semioparticipativa, Rosa e Matos [20] conceberam o SPIDe, um processo para o design de interação semioparticipativo. Desde a sua formulação, o SPIDe tem sido reexaminado e expandido por outros pesquisadores $[20,18,19]$, inclusive com enfoque à elicitação de requisitos.

\section{SPIDe}

O SPIDe é um processo semioparticipativo para o design de interação de artefatos computacionais [20]. Sob a perspectiva da semioparticipação, proposta por Baranauskas [3], o SPIDe tem a base teórico-conceitual fundamentada na Engenharia Semiótica (EngSem) [12] - diferentemente da proposta de Baranauskas [3], que faz uso da Semiótica Organizacional como fundamento teórico-conceitual - e princípios e técnicas do Design Participativo (DP) [22].

O SPIDe implementa o Design Centrado na Comunicação (DCC), uma prática da EngSem que considera o design de interação como um processo de manipulação de mensagens. O DCC divide o processo de design de interação em três etapas: (i) análise de contexto, (ii) engenharia de interface e (iii) avaliação. No SPIDe, cada uma dessas etapas é composta por técnicas do DP e apesar de serem apresentadas sequencialmente, conforme a Figura 1, elas podem ser (re)visitadas quantas vezes for necessário. O SPIDe, enquanto processo metodológico, sugere sempre o uso das mesmas técnicas e etapas. Todavia, isso não significa que não seja possível refazer a aplicação de uma ou mais técnicas ou etapas, caso seja necessário [20].

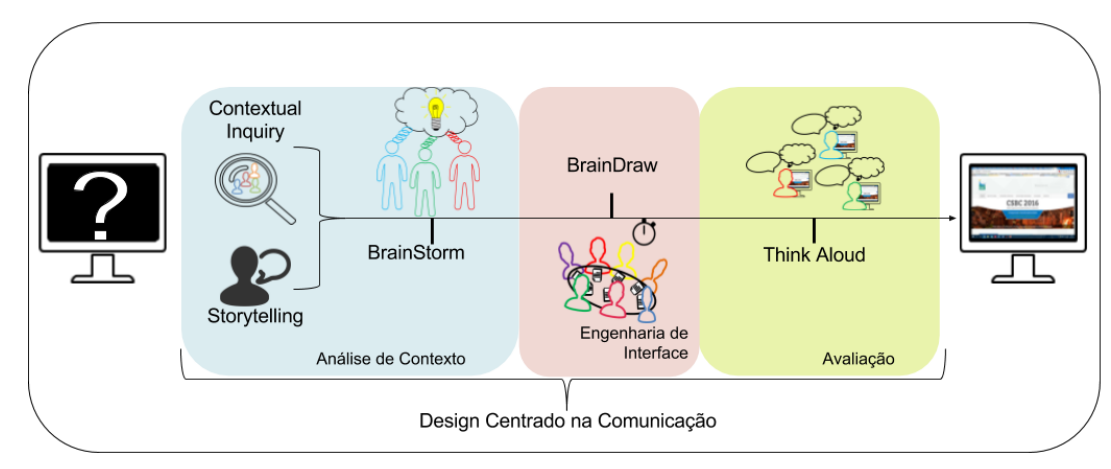

Figura 1. SPIDe [18].

A primeira versão do SPIDe ( $c f .[20])$ foi composta por quatro técnicas de DP (contextual inquiry, brainstorm, braindraw e think-aloud), tendo sido aplicada no domínio educacional, com estudantes de ensino fundamental e professores para 
o redesign de interação da rede socioeducacional TecCiencia. O SPIDe considera o paradigma de design por/para todos. Na sua aplicação inicial, Rosa e Matos [20] se dedicaram a evidenciar aspectos culturais no design de interação. Para os pesquisadores, o SPIDe pode considerar esses aspectos devido a sua característica semioparticipativa.

A pesquisa apresentada por Pita et al. [18] continuou o desenvolvimento do SPIDe. Aplicou-se a análise de contexto do SPIDe no domínio de mobilidade urbana, como usuários com deficiência visual, também a partir da perspectiva de design por/para todos. A pesquisa resultou na primeira modificação do SPIDe. Pita et al. [18] sugeriram a adição da técnica storytelling. A sugestão foi realizada devido alguns usuários se sentirem constrangidos pela aplicação do contextual inquiry. Desse modo, conforme a sugestão dos autores, deve-se escolher entre a aplicação do contextual inquiry ou do storytelling durante o (re)design de interação.

De acordo com Rosa e Matos [20] e Pita et al. [18], por meio do SPIDe é possível gerar requisitos para o (re)design de interação (humano-computador) considerando aspectos culturais, a participação de crianças, adultos e deficientes visuais no domínio educacional e de mobilidade urbana.

A partir desses estudos sobre o SPIDe [20,18], foi possível identificar a possibilidade de utilização do SPIDe quando o projeto: (i) possuir diversos perfis de usuários, sejam por diferenças culturais, cognitivas, motoras, sociais e, inclusive, hierárquicas; (ii) quando tiver (ou ainda não tiver) definição clara dos problemas a serem tratados; (iii) quando o contato do engenheiro de requisitos com o usuário for para além da aplicação de questionários ou da construção de personas.

Rosa et al. [19] também seguiram expandindo o SPIDe; todavia, sob a perspectiva de necessidades e desejos (i.e. requisitos) dos usuários. Os pesquisadores investigaram o uso das técnicas indicadas no SPIDe em atividades de elicitação de requisitos. No estudo, os pesquisadores realizaram uma revisão sistemática da literatura sobre o uso das técnicas que compõem o SPIDe aplicadas à ER. Foram encontradas em produções científicas somente o uso das técnicas storytelling e brainstorm aplicadas à ER. Todavia, não foi possível identificar quais tipos de requisitos são gerados por essas técnicas, nem foram encontradas aplicações do contextual inquiry, braindraw e think-aloud, deixando lacunas para a continuação da pesquisa.

\section{Metodologia}

Dando sequência a pesquisa sobre o SPIDe, este artigo apresenta um estudo com o objetivo de experimentar se com a aplicação do SPIDe é possível elicitar requisitos. Para isso, foi conduzido um estudo experimental com um problema real, para a concepção de um Ambiente Virtual de Aprendizagem (AVA) para a Biblioteca Estadual Epifânio Dória, em Aracaju/SE. A concepção e desenvolvimento do AVA foi realizada pela equipe do projeto de extensão universitária intitulado "Uma proposta de Ambiente Virtual de Aprendizagem para disseminação 
da inclusão digital na sociedade Sergipana", vinculado à Universidade Federal de Sergipe.

O SPIDe em sua concepção, apresentado em um artigo anterior [20], foi direcionado ao (re)design de interação de softwares educacionais. Em um estudo posterior [18] foi experimentada a aplicação do SPIDe com usuários com deficiência visual ou cegos. Por sua vez, este artigo continua o desenvolvimento do SPIDe no contexto de softwares educacionais, mas focalizando na elicitação de requisitos.

Este estudo foi realizado in-vivo, no laboratório de informática da Biblioteca. Participaram da pesquisa o coordenador de cursos, um professor dos cursos presenciais e seis alunos dos cursos presenciais já oferecidos pela Biblioteca. Os participantes foram convidados por conveniência, tinham entre 16 e 46 anos de idade, a maioria são moradores de comunidades carentes e, alguns deles, possuem limitações para a utilização de ferramentas computacionais. Antes da aplicação do estudo, foi solicitada a assinatura dos termos de consentimento livre e esclarecido aos participantes. É importante destacar também que a aplicação do estudo foi realizada por alunos do curso de Bacharelado em Sistemas de Informação da Universidade Federal de Sergipe, que assumiram o papel de engenheiro de requisitos.

A primeira técnica aplicada, conforme previsto pelo SPIDe, foi o storytelling ${ }^{5}$. Nessa aplicação do SPIDe, optou-se por utilizar o storytelling devido a existência de experiências anteriores com sucesso ao utilizar essa técnica, conforme relatos encontrados na literatura [19]. A técnica foi aplicada por uma engenheira de requisitos e contou com a participação do coordenador do curso, um professor e seis alunos, individualmente. Os participantes foram convidados a narrar seu trabalho/atividade na Biblioteca, explicando suas dificuldades e necessidades em relação ao produto a ser desenvolvido. Por meio do storytelling foi possível coletar áudios com histórias contadas por cada participante.

Logo depois, o brainstorm foi aplicado em grupo, com os mesmos participantes do storytelling. Durante a aplicação da técnica, notas autoadesivas foram distribuídas para todos os participantes. Eles escreveram ideias nessas notas sob moderação da engenheira de requisitos. A engenheira de requisitos questionou sobre as atividades realizadas pelos participantes e sugestões que foram dadas para a construção do software durante a aplicação da técnica de storytelling. As notas foram apresentadas em um quadro para que todos os participantes tivessem acesso às ideias sugeridas e permitissem que novas ideias fossem produzidas.

Com a identificação do contexto e principais necessidades dos participantes em relação à construção do AVA, a técnica braindraw foi aplicada para identificar novos requisitos e permitir que os participantes criem desenhos relacionados à interface que será construída pela equipe de desenvolvimento. O mesmo conjunto de participantes do storytelling e do brainstorm foi convidado; todavia, o coordenador não compareceu.

\footnotetext{
${ }^{5}$ De acordo com Pita et al. [18], para iniciar o processo deve-se escolher entre o contextual inquiry e o storytelling.
} 
Para aplicação braindraw foram utilizados os seguintes materiais: lápis grafite e colorido, borrachas, canetas, papel A4 e cronômetro. Os participantes ficaram próximos uns dos outros e cada um recebeu uma folha de papel, conforme exemplifica a Figura 2. Cada participante fez um desenho expressando como ele acha que a interface do software deve ser desenvolvida, considerando as necessidades relatadas por eles durante a análise de contexto. Ao término do intervalo de dois minutos, cada desenho foi passado ao participante do lado esquerdo que, por sua vez, repetiu o procedimento, completando o desenho dado a ele. Esse processo se seguiu até todos os desenhos retornarem a quem iniciou, de modo que todos contribuíssem em todos os desenhos. Com isso foi possível reunir desenhos que representassem a fusão de ideias de todos os participantes, conforme indicado por Muller, Haslwanter e Dayton [15].

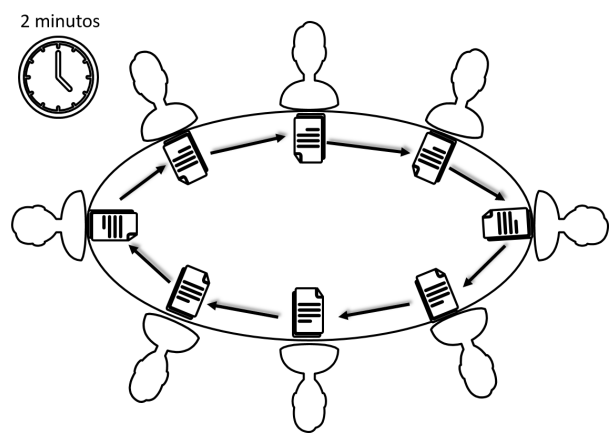

Figura 2. Disposição durante braindraw [20].

Por fim, o think-aloud foi aplicado, com o mesmo conjunto de sujeitos participantes do braindraw. Para auxiliar no registro da aplicação, uma câmera foi posicionada em frente ao monitor do participante com o objetivo de registrar suas ações e fala. Esta técnica foi realizada utilizando os protótipos produzidos a partir dos resultados do braindraw e das necessidades identificadas com a aplicação das técnicas storytelling e brainstorm. Os participantes foram encorajados pela engenheira de requisitos a usar o protótipo, navegando nas interfaces e expressando suas opiniões durante a interação.

\section{Resultados}

A análise dos dados foi realizada a partir dos documentos resultantes da aplicação das técnicas storytelling, brainstorm, braindraw e think-loud durante o processo de elicitação de requisitos. 


\subsection{Storytelling}

A engenheira de requisitos do projeto conseguiu descobrir 17 requisitos a partir da aplicação do storytelling. Entre os requisitos encontrados, 14 eram requisitos funcionais (RF) e 3 requisitos não funcionais (RNF), do quais 2 foram classificados como requisitos não funcionais de interação e 1 de segurança. Estes requisitos são apresentados na Tabela 1.

Tabela 1. Resultado do storytelling

\begin{tabular}{lccccc}
\hline ID & Descrição & Tipo & ID & Descriação & Tipo \\
\hline RF001 & Manter curso & RF & RF010 & Acesso as notas do curso & RNF \\
\hline RF002 & Manter Professor & RF & RF011 & Emissão de certificados & RF \\
\hline RF003 & Manter aluno & RF & RF012 & Frequência dos alunos & RF \\
\hline RF004 & Acesso aos cursos & RF & RF013 & Lançamento de frequência & RF \\
\hline RF005 & Área de sugestões & RF & RF014 & Relatórios específicos & RF \\
\hline RF006 & Área de Bate Papo & RF & RNF001 & Acesso web & RNF \\
\hline RF007 & Fórum dos cursos & RF & RNF002 & Interface limpa e clara & RNF \\
\hline RF008 & Materiais dos cursos & RF & RNF003 & Login e senha & RNF \\
\hline RF009 Sala de videoconferência & RF & & &
\end{tabular}

É importante destacar que durante o storytelling o usuário foi convidado a contar suas histórias e experiências relacionadas ao seu trabalho/atividade na biblioteca. Para isso os usuários fizeram uso do seu conhecimento prévio para comunicar o que era desejável. Esse conhecimento prévio está relacionado aos seus conhecimentos subjetivos sobre como as coisas acontecem na biblioteca para a promoção dos cursos, sejam eles presenciais ou a distância. Portanto, características culturais, emocionais, cognitivas e sociais puderam ser reunidas e exploradas por meio da Engenharia Semiótica. Um exemplo é a fala de um dos usuários durante o storytelling ${ }^{6}$ :

"Tem muita gente que não tem horário, tipo poderia não ter horário nenhum, onde a pessoa poderia estudar de madrugada, dependendo de sua força de vontade, e também cada um poderia ter seu perfil que querendo ou não tem coisas que só tiramos dúvidas com o professor, que ai eu poderia conversar com minha professora. Vamos dizer assim, tem um chat específico para uma pessoa, [...]. Então, também poderia ter também o boletim, a pessoa poderia tirar o certificado."

Nessa fala identificou-se a preocupação com pessoas sem horário disponível para realizar um curso presencial. Isso demonstra a aptidão do SPIDe em alcançar características culturais e sociais dos usuários. Além disso, é possível identificar alguns requisitos em sua fala: RF006, RF010, RF011.

\footnotetext{
${ }^{6}$ Os áudios do storytelling foram transcritos para a análise
} 


\subsection{Brainstorm}

Com a aplicação da técnica de brainstorm identificou-se sete RF e um RNF, conforme disposto na Tabela 2. Entre os requisitos identificados a partir dessa técnica, apenas o requisito RF015 não havia sido identificado com a aplicação do storytelling.

Tabela 2. Requisitos elicitados a partir do brainstorm.

\begin{tabular}{ccc}
\hline ID & Descrição & Tipo \\
\hline RF004 & Acesso aos cursos & RF \\
\hline RF006 & Área de bate papo & RF \\
\hline RF007 & Fórum do curso & RF \\
\hline RF008 & Materiais do curso & RF \\
\hline RF009 & Sala de videoconferência & RF \\
\hline RF010 & Acesso as notas do curso & RF \\
\hline RF015 & Notificar aluno & RF \\
\hline RNF002 & Interface limpa e clara & RNF \\
\hline
\end{tabular}

Embora tenha sido possível coletar requisitos que já haviam sido especificados com a aplicação do storytelling, o brainstorm possibilitou a identificação de atributos importantes em relação aos requisitos previamente identificados. Alguns desses atributos foram coletados de anotações, como a solicitação de nome, idade, endereço para o cadastro; e a necessidade de ocultação do endereço, disponível somente para pessoas previamente autorizadas . A Figura 3 mostra exemplos de notas resultantes dessa aplicação.

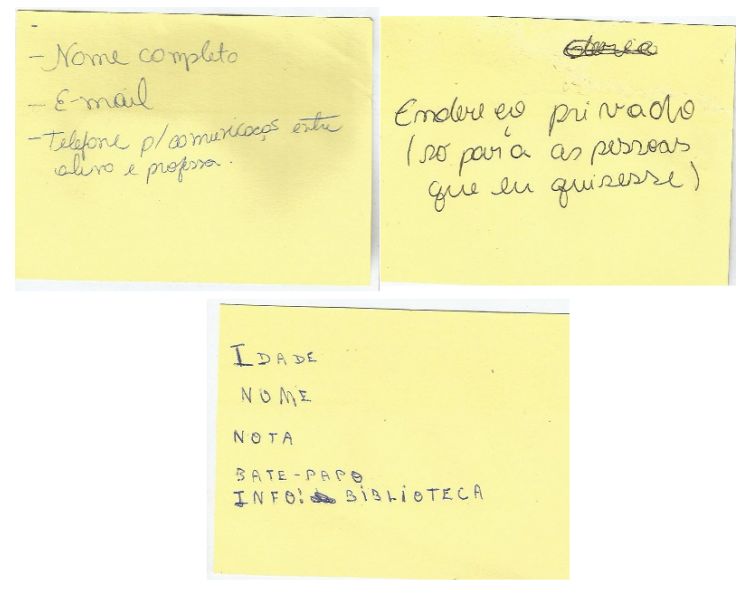

Figura 3. Notas do brainstorm. 


\subsection{Braindraw}

A partir da aplicação do braindraw foi possível construir protótipos de interfaces ${ }^{7}$ considerando os diferentes perfis de participantes e seus respectivos desejos e necessidades a partir dos desenhos da Figura 4. Os protótipos construídos a partir dos desenhos também contêm requisitos elicitados com a aplicação de técnicas de storytelling e brainstorm. Além de possibilitar a construção de protótipos contendo os requisitos já elicitados, os protótipos também permitiram representar a interface que será desenvolvida de acordo com a proposta dos participantes e considerando seus sistemas de significação.

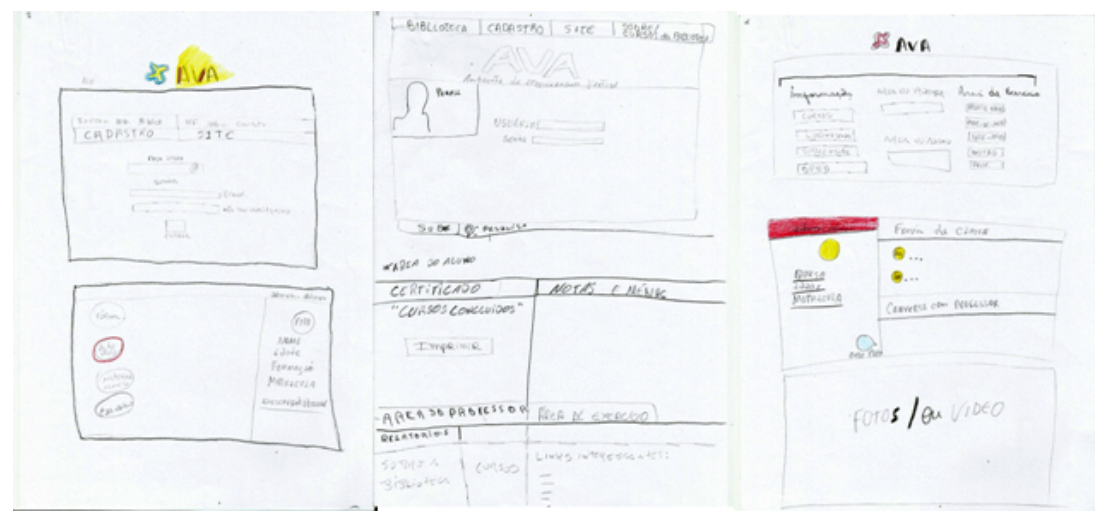

Figura 4. Desenhos resultantes do braindraw.

Por meio dos protótipos também identificou-se alguns novos requisitos, tais como: upload de vídeos e fotos, sugestões de melhorias para a biblioteca.

\subsection{Think-aloud}

Com a aplicação do think-aloud foi possível validar com o usuário os requisitos elicitados com a aplicação de técnicas anteriores utilizando protótipos desenvolvidos. A análise foi realizada sobre os documentos produzidos e de acordo com áudios coletados durante a aplicação desta técnica, tais como: "[...] está limpo, não tem muita informação. Está fácil de ser utilizado" e "Tudo ok, não mudaria nada". Frases semelhantes a essas permitiram concluir que os requisitos elicitados e implementados nos protótipos estavam de acordo com os desejos e necessidades dos participantes.

Encontrou-se também sugestões para melhorias em relação ao design de interação do AVA, uma vez que problemas em relação ao layout do software foram sinalizados por alguns participantes. Isso indicou a necessidade de mudanças

$\overline{7}$ cf. https://marvelapp.com/673fjf1/screen/31157836 
relacionadas à disposição das funções. O trecho abaixo, relatado por um dos participantes ao experimentar a página inicial do aluno, reflete a solicitação de ajustes relacionados à interface produzida.

"Aqui eu achei que ficou muitos links [referente a área com dados do aluno], poderia ter a nossa foto aqui e ter só editar dados e ter só notas cursos e certificados. Essas outras partes, nome, email, informação... tal tal tal é no link de editar dados. Tiraria tudo isso e ficaria só o editar dados. Ficaria mais limpo".

\section{Considerações Finais}

Este artigo apresentou os resultados de um estudo com o objetivo de identificar a possibilidade de uso do SPIDe para elicitação de requisitos de software. Considerando o desafio de comunicação nos processos de engenharia de requisitos [17], o SPIDe pode contribuir ao tornar os usuários co-autores do software a ser desenvolvido, a partir da perspectiva teórico-conceitual da Engenharia Semiótica e princípios e técnicas do Design Participativo.

Nesse sentido, o SPIDe, inicialmente um processo semioparticipativo para design de interação, foi aplicado na elicitação de requisitos de um Ambiente Virtual de Aprendizagem para uso de uma biblioteca pública. Com o SPIDe, orientado pelo design centrado na comunicação foi possível conhecer os usuários; sugestões, necessidades, desejos e críticas; produzir protótipos de interfaces; e avaliar esses protótipos, conforme descrito por Rosa e Matos [20] e Pita et al. [18].

Considerando que os usuários participaram da aplicação do SPIDe, é possível pressupor que aspectos subjetivos, tais como cultural, valores, características físico-motoras, cognitivas foram valorizados, conforme indicado por Rosa e Matos [20]. Além disso, a engenheira de requisitos também participou de todo o processo, elicitando e mediando a aplicação das técnicas, além de analisar os resultados e descrever os requisitos descobertos.

A aplicação do SPIDe permitiu aos usuários comunicar seus desejos e necessidades à engenheira de requisitos, de forma implícita ou explicita. Por sua vez, também foi possível à engenheira de requisitos identificar como os usuários desejam interagir com o software, como eles percebem a interface, quais problemas de interação e de projeto devem ser evitados, restrições e atributos que o software deve considerar.

Nesse sentido, foi possível identificar evidências que por meio do SPIDe é possível elicitar requisitos, permitindo aos usuários tornarem-se co-autores do projeto de software. Apesar de o SPIDe ser um processo inicialmente para o design de interação, os requisitos elicitados por meio de sua aplicação não foram apenas relacionados a requisitos de interação ou não funcionais, mas também a requisitos funcionais.

Há algumas ameaças à validade deste estudo, dentre elas, destacam-se: a falta da participação de um professor do ensino a distância (o professor participante 
era de cursos presenciais); aplicação SPIDe por uma estudante do curso de Bacharelado em Sistemas de Informação, com experiência mediana na elicitação de requisitos (apesar de estudos apontarem que há apenas poucas diferenças na participação de estudantes ou profissionais em pesquisas científicas); a contribuição limitada pelos dados, por causa do perfil dos participantes.

Como trabalhos futuros, estudos mais aprofundados, mais metódicos do ponto de vista da seleção dos participantes e das técnicas de análise dos dados, serão realizados para aprofundar o nível em que os requisitos são elicitados. Além disso, será realizada uma comparação entre o SPIDe e outros processos de elicitação de requisitos, como: Design Thinking [23], JAD [9] e MUST [7]. Posteriormente, a contribuição do SPIDe para a Engenharia de Requisitos Ágeis será investigada, considerando a relação custo-benefício de cada técnica, o tempo gasto e o esforço em suas aplicações, tópicos que ainda são desafios em abertos para o SPIDe e estão sendo avaliados. Espera-se abordá-los em trabalhos futuros.

\section{Referências}

1. Arantes, F. L.: Requirements Engineering of a Web Portal Using Organizational Semiotics Artifacts and Participatory Practices. International Journal of Computer Science and Information Technology 5(2), 131-146 (2013). https://doi.org/10.5121/ijcsit.2013.5212

2. Arantes, F. L.: Organizational Semiotics and Participatory Design to Requirements Elicitation - A Case Study. In: Proceedings of VII Brazilian Symposium on Information Systems, pp. 322-333. SBC (2011)

3. Baranauskas, M. C. C.: Social awareness in HCI. Interactions 21(4), 66-69 (2014). https://doi.org/10.1145/2621933

4. Baranauskas, M. C. C.: O modelo semioparticipativo de design. In: Baranauskas, M. C. C., Martins, M. C., Valente, J. A. (eds.) Codesign de Redes Digitais: tecnologia a serviço da inclusão social. Penso (2013)

5. Baranauskas, M. C. C. Schimiguel, J. Simoni, C. A. C. Medeiros, C. B.: Guiding the Process of Requirements Elicitation with a Semiotic-based Approach - A Case Study. In: Proceedings of the 11th International Conference on Human-Computer Interaction, pp. 100-111. Lawrence Erlbaum Associates (2005)

6. Barraza, Í. D. Zedepeta, V. V.: Factores sociales y humanos que afectan el proceso de educción de requerimientos: una revisión sistemática. RISTI - Revista Ibérica de Sistemas e Tecnologias de Informação 24(10), 69-83 (2017). https://doi.org/10.17013/risti.n.69-83

7. Bødker, K., Kensing, F., Simonsen, J. Participatory Design in Information Systems Development. In: Isomäki, H., Pekkola, S. (eds.) Reframing Humans in Information Systems Development. Springer London (2011). https://doi.org/10.1007/9781-84996-347-3_7

8. Bourque, P., Fairley, R. E.: Guide to the Software Engineering - Body of Knowledge. 3nd edn. IEEE Computer Society (2014)

9. Coughlan, J. Macredie, R. D.: Effective communication in requirements elicitation: A comparison of methodologies. Requirements Engineering 2(7), 47-60 (2002). https://doi.org/10.1007/s007660200004

10. Da Costa, H. F. D. Pimentel, J. H. C.: O Papel da Comunicação na Elicitação de Requisitos - Entrevistas e Brainstorming. Revista de Engenharia e Pesquisa Aplicada 4(2), 97-109 (2017). https://doi.org/10.25286/repa.v2i4.845 
11. Das, V. V: Involvement of users in software requirement engineering. In: Proceedings of 10th International Conference on Information Technology, pp. 230-233. IEEE Computer Society (2007)

12. De Souza, C. S.: The Semiotic Engineering of Human-Computer Interaction. MIT Press (2005)

13. Eco, U.: A Theory of Semiotics. Indiana University Press (1976)

14. Lamsweerde, A. van: From System Goals to UML Models to Software Specifications. Wiley (2008)

15. Muller, M. J. Haslwanter, J. H. Dayton, T.: Participatory Practices in the Software Lifecycle. In: Helander, M. G. Landauer, T. K. Prabhu, P. V. (eds.) Handbook of Human-Computer Interaction, pp. 255-297. Elsevier (1997)

16. Nuseibeh, B. Easterbrook, S.: Requirements engineering: a roadmap. In: Proceedings of the conference on The future of Software engineering - ICSE'00, pp. 35-46. ACM Press (2000). https://doi.org/10.1145/336512.336523

17. Pa, N. C. Zin, A. M.: Requirement Elicitation: Identifying the Communication Challenges between Developer and Customer. International Journal on New Computer Architectures and Their Applications (IJNCAA) 1(2), 371-383 (2011)

18. Pita, G. L. Zabot, D. Rosa, J. Matos, E.: Adapting the SPIDe to Include Visually Impaired Users in Interaction Design. In: Proceedings of the XVI Brazilian Symposium on Human Factors in Computing Systems - IHC 2017, pp. 1-4. ACM Press (2017). https://doi.org/10.1145/3160504.3160579

19. Rosa, J. Silva, F. S. Silva, G. J. F. Matos, E.: Applying SPIDe's Techniques in Requirements Engineering : a sistematic review. Computing and System Journal $\mathbf{7}(2), 290-303$ (2017)

20. Rosa, J. C. S. Matos, E.: Semio-Participatory Framework for Interaction Design of Educational Software. In: Proceedings of the 15th Brazilian Symposium on Human Factors in Computer Systems - IHC'16, pp. 1-10. ACM Press (2016). https://doi.org/10.1145/3033701.3033734

21. Santaella, L.: O que é Semiótica. Brasiliense (1990)

22. Simonsen, J., Robertson, T.: Routledge International Handbook of Participatory Design. Taylor \& Francis (2012). https://doi.org/10.4324/9780203108543

23. Souza, A. F. Ferreira, B. M. Conte, T.: Aplicando Design Thinking em Engenharia de Software : um Mapeamento Sistemático. In: Proceedings on Ibero-American Conference on Software Engineering: Experimental Software Engineering Latin America Workshop (CIbSE-ESELAW), pp. 719-732. (2017)

24. Wagner, S. Fernández, D. M. Felderer, M. Vetró, A. Kalinowski, M. Wieringa, R. Pfahl, D. Conte, T. Christiansson, M. Greer, D. Lassenius, C. Männistö, T. Nayebi, M. and Oivo, M. Penzenstadler, B. Prikladnicki, R. Ruhe, G. Schekelmann, A. Sen, S. Spínola, R. Tuzcu, A. de la Vara, J. L. Winkler, D.: Status Quo in Requirements Engineering: A Theory and a Global Family of Surveys. http://arxiv.org/abs/1805.07951 Last accessed 17 Jul 2018

25. Zin, A. M. Pa, N. C.: Measuring Communication Gap in Software Requirements Elicitation Process. In: Proceedings of the 8th International Conference on Software Engineering, Parallel and Distributed Systems, pp. 66-71. World Scientific and Engineering Academy and Society (2009) 